\title{
ANALOG LOW-VOLTAGE CURRENT-MODE IMPLEMENTATION OF DIGITAL LOGIC GATES
}

\author{
MUHAMMAD TAHER ABUELMA'ATTI \\ King Fahd University of Petroleum and Minerals, Box 203, Dhahran 31261, Saudi Arabia
}

(Received 3 September 2002; In final form 17 November 2002)

\begin{abstract}
In this letter a new technique is introduced for implementing the basic logic functions using analog current-mode techniques. By expanding the logic functions in power series expressions, and using summers and multipliers, realization of the basic logic functions is simplified. Since no transistors are working in saturation, the problem of fan-out is alleviated. To illustrate the proposed technique, a circuit for simultaneous realization of the logic functions NOT, OR, NAND and XOR is considered. SPICE simulation results, obtained with $3 \mathrm{~V}$ supply, are included.
\end{abstract}

Keywords: Digital logic circuits; Translinear principle

\section{INTRODUCTION}

Mixed analog/digital electronic circuits are becoming increasingly important. Digital electronic circuits are mostly designed in CMOS technology. To be able to integrate the digital and analog parts on to one chip, high-performance analog CMOS circuits are required [1] and a large number of mixed analog/digital VLSI integrated circuits realized in state-of-the-art digital CMOS technologies are now available [2]. In fact the emergence of ICs incorporating mixed analog and digital functions on a single chip has led to an advanced level of analog design [3]. Of particular interest here is the current-mode approach for designing analog ICs. It is well known that current-mode analog signal processing offers some important speed advantages over the traditional voltage-mode signal processing [4].

At present, current-mode implementations are available for a wide range of analog electronic circuits including $\mathrm{A} / \mathrm{D}$ and $\mathrm{D} / \mathrm{A}$ converters, continuous time filters, neural-networks, sampled data filters and microwave and optical systems. This raises the following question: can digital ICs be realized using current-mode analog techniques? In fact analog-based realizations of digital logic circuits may result in avoiding the traditional problems of fan-in and fan-out, inherent in digital-based implementations, less complexity, low-voltage as well as higher speed of operation [5]. In an attempt to answer this question, the translinear principle [6] has been used to realize a digital inverter circuit [7, 8] and a bistable element [9]. Survey of the literature yields no systematic approach for designing digital logic circuits using analog current-mode techniques. This paper is an attempt to present such an approach. 


\section{POWER SERIES REPRESENTATION OF LOGIC FUNCTIONS}

Using their truth tables, it is easy to show that the input-output relations of the basic digital logic functions can be expressed as [5]:

$$
z=1-x
$$

for the NOT operation,

$$
z=x * y
$$

for the AND operation,

$$
z=x+y-x * y
$$

for the OR operation, and

$$
z=x+y-2 x * y
$$

for the XOR operation. In Eqs. (1)-(4) the signs,+- and $*$ carry their normal mathematical meanings, that is add, subtract and multiply respectively. Using Eqs. (2)-(4) in combination with Eq. (1), the digital-logic functions NAND, NOR and XNOR can be realized.

Analog implementation of the basic logic functions (1)-(4) requires analog multipliers, inverters and summers. Using a modified version of the four-quadrant multiplier reported in Ref. [10], voltage-mode analog implementation of two-input AND, NOT and OR functions has been reported [5]. These implementations, however, are built around voltage-mode operational amplifiers and analog switches, use a large number of resistors and require relatively large supply voltages. This paper presents alternative current-mode analog implementations of the digital logic functions. Using no resistors, except for realizing constant current-sources, no switches, no operational amplifiers and only a small number of transistors, the proposed implementations require low supply voltages and are very attractive for integration.

\section{PROPOSED CIRCUIT}

The proposed circuit is shown in Figure 1. Transistors $\mathrm{Q}_{7}-\mathrm{Q}_{13}$ form a set of current mirrors for the input current $x$, and transistors $\mathrm{Q}_{14}-\mathrm{Q}_{17}$ form a set of current mirrors for the input current $y$. Transistors $\mathrm{Q}_{4}-\mathrm{Q}_{6}$ form current mirrors for the collector current of transistor $\mathrm{Q}_{4}$. Transistors $\mathrm{Q}_{1}-\mathrm{Q}_{4}$ form an npn current mirror with controlled gain [6]. Using the translinear principle [6], it is easy to show that the collector current of the transistor $\mathrm{Q}_{4}$ can be expressed as

$$
I_{C 4}=x * y
$$

With the emitter-area of transistor $\mathrm{Q}_{6}$ equal to twice the emitter-area of transistors $\mathrm{Q}_{4}$ and $\mathrm{Q}_{5}$, the collector currents in transistors $\mathrm{Q}_{5}$ and $\mathrm{Q}_{6}$ can be expressed as

$$
I_{C 6}=2 I_{C 5}=2 x * y
$$




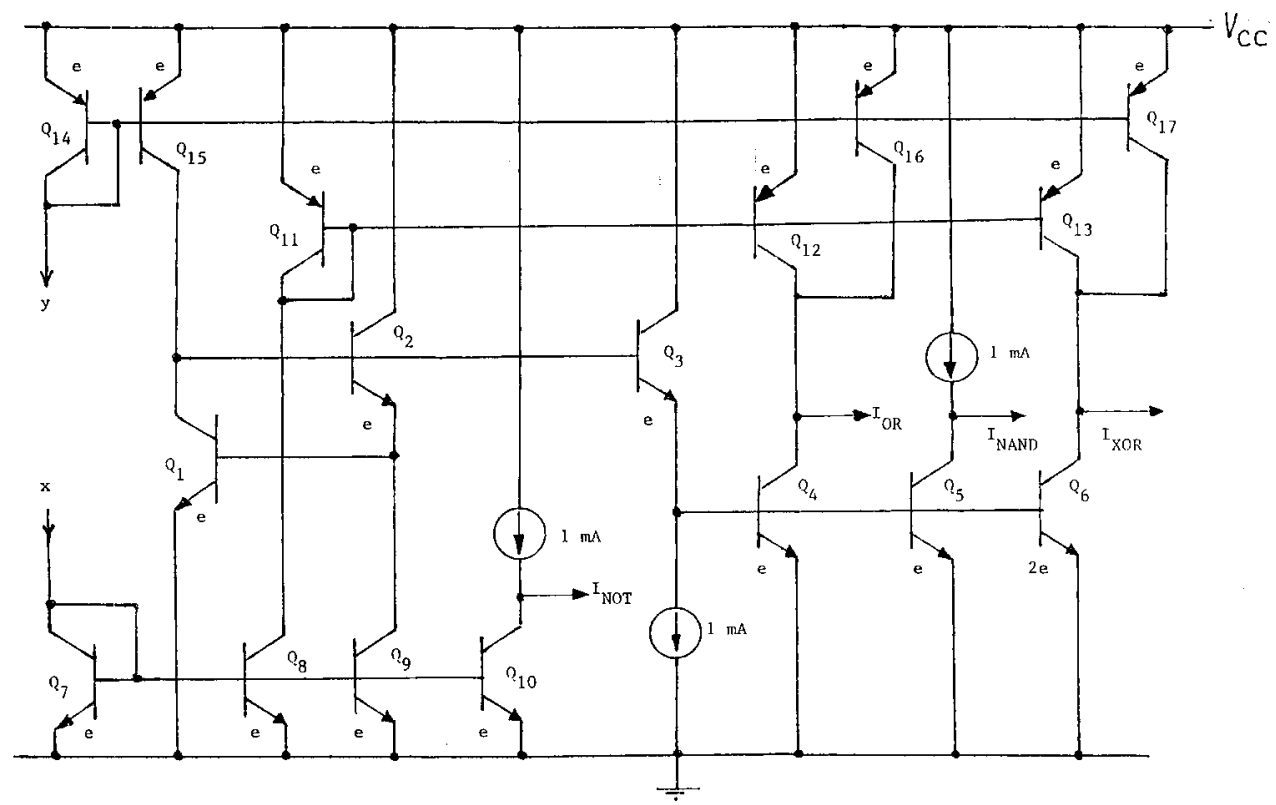

FIGURE 1 Proposed implementation of the NOT/OR/NAND/XOR digital logic functions using analog techniques.

Using Eqs. (5) and (6) it is easy to verify that the output currents $I_{\mathrm{NOT}}, I_{\mathrm{OR}}, I_{\mathrm{NAND}}$ and $I_{\mathrm{XOR}}$ realize the logic functions NOT, OR, NAND and XOR given by Eqs. (1)-(4). Realization of the logic functions NOR, AND and XNOR is a straightforward extension of the implementations of Figure 1.

\section{SIMULATION RESULTS}

The proposed circuit of Figure 1 was simulated using the $2 \mathrm{~N} 2222$ npn transistors and the $2 \mathrm{~N} 2905$ pnp transistors and supply voltage $V_{\mathrm{CC}}=3 \mathrm{~V}$. The results obtained from the NOT, OR, NAND and XOR operations are shown in Figure 2. In Figure 2 the currents are sensed using $1 \mathrm{k} \Omega$ load resistances. With $\beta_{F}=105$ for the npn transistor and 325 for the pnp transistor and with $V_{A F}=98.5$ for the npn transistor and 139.0 for the pnp transistors, it appears that, using general purpose transistors, the results shown in Figure 1 are in excellent agreement with the theory presented in Eqs. (1)-(4).

\section{CONCLUSION}

Starting from the truth tables of logic functions, it is possible to obtain power series expansions of the basic logic functions. In these expansions the signs,+- and $*$ carry their traditional mathematical meanings, that is add, subtract and multiply respectively. Thus, using these expansions, it is possible to implement the basic logic functions using analog currentmode techniques. In this letter a simple circuit, using $3 \mathrm{~V}$ supply voltage, has been presented for simultaneous realization of the basic logic functions NOT, OR, NAND and XOR using techniques widely used in realizing analog current-mode circuits. The proposed circuit 


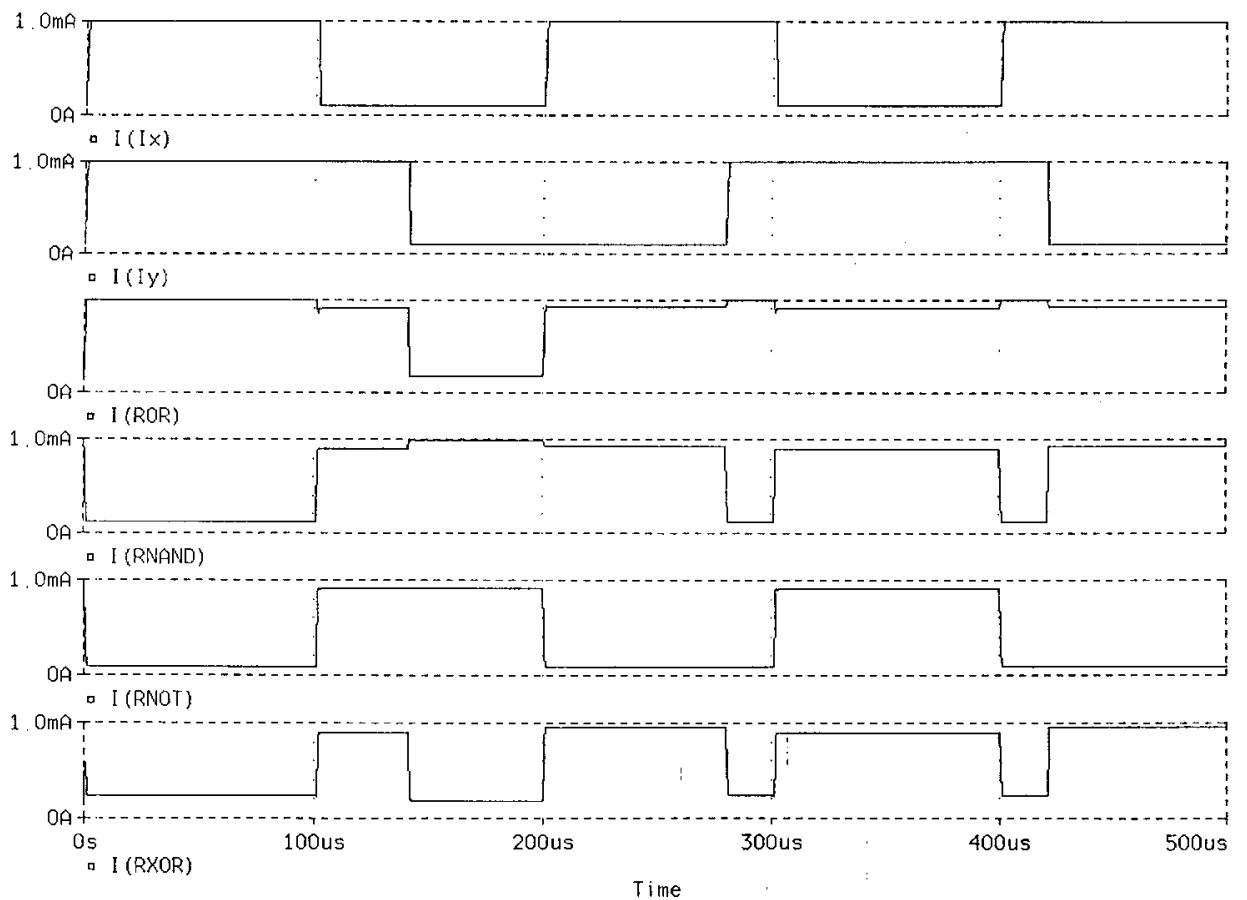

FIGURE 2 Results obtained from the proposed circuit of Fig. 1 with DC supply voltage $=3 \mathrm{~V}$.

can be easily extended to realize the functions NOR, AND and XNOR. Moreover, extension of the proposed circuit to realize basic logic functions with number of inputs greater than two is also straightforward. Since the output transistors are no longer requested to operate in the saturation region, the problem of fan-out is alleviated.

It is expected that using this approach for implementing more sophisticated logic circuits, for example encoders/decoders, will result in simpler and faster realizations. Finally, by realizing analog and digital circuits using the same basic building blocks, it is expected that simple design procedures for mixed analog/digital circuits and systems may emerge.

\section{References}

[1] Ismail, M. and Fiez, T. (1994). Analog VLSI Signal and Information Processing. McGraw-Hill, NY.

[2] Laker, K. R. and Sansen, W. M. C. (1994). Design of Analog Integrated Circuits and Systems. McGraw-Hill, NY.

[3] Toumazou, C., Lidgey, J. and Haigh, D. (1990). Analog IC Design: The Current Mode Approach. Peter Peregrinus, London.

[4] Allen, P. E. (1990). Future of analogue integrated circuit design. In: Toumazou, C., Lidgey, J. and Haigh, D. (Eds.), Analog IC Design: The Current Mode Approach. Peter Peregrinus, London.

[5] Enab, Y. M. I. and Zaki, F. W. (1993). Power series representation of logical functions and its application to error detection and error correction codes. Mansoura Engineering Journal, 18(3), E.1-E.12.

[6] Gilbert, B. (1990). Current-mode circuits from a translinear viewpoint. A tutorial. In: Toumazou, C., Lidgey, J. and Haigh, D. (Eds.), Analog IC Design: The Current Mode Approach. Peter Peregrinus, London.

[7] Kemp, A. J. (1983). Translinear logic - A new technique in bipolar technology. Electronics Letters, 19, 349-350.

[8] Kemp, A. J. (1984). Evaluation of translinear logic. Electronics Letters, 20, 413-414.

[9] Seevinck, E. (1978). Application of the translinear principle in digital circuits. IEEE Journal of Solid-State Circuits, SC-13, 528-530.

[10] Khachab, N. and Ismail, M. (1991). A nonlinear CMOS analog cell for VLSI signal information processing. IEEE Journal of Solid-State Circuits, 26, 1689-1699. 

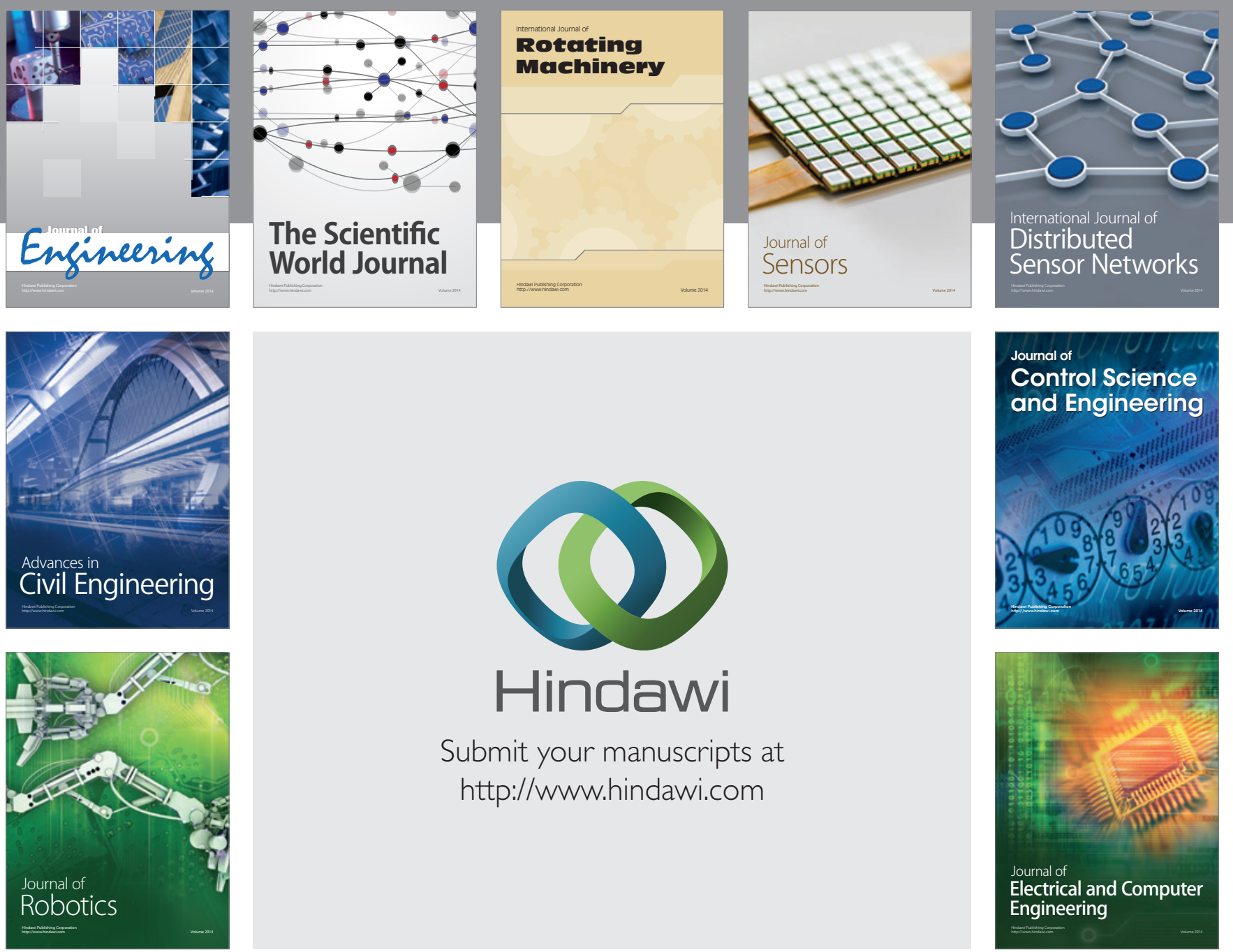

Submit your manuscripts at

http://www.hindawi.com
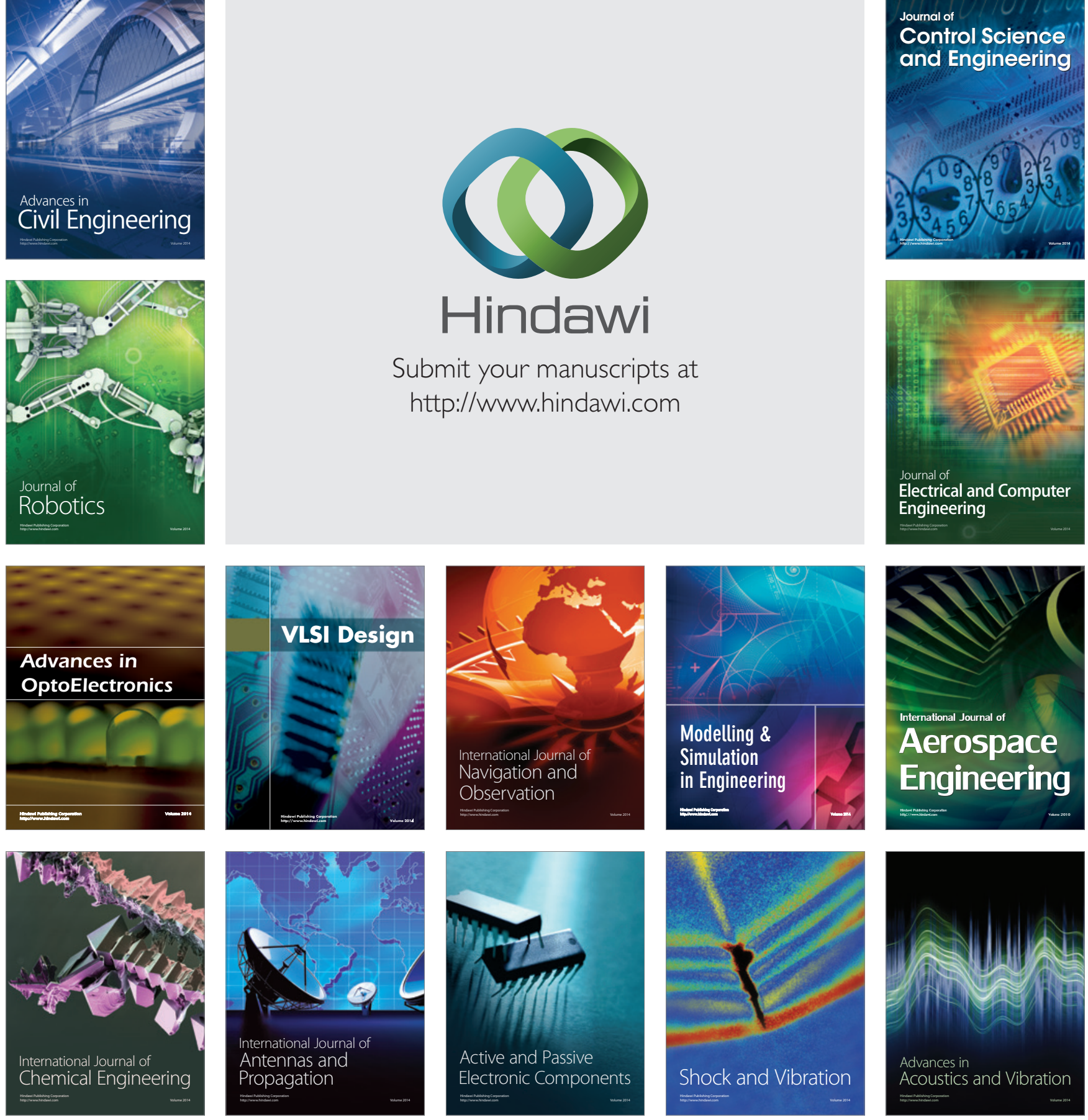